\title{
RUOLI PRAGMATICI DI «Sİ»: Modello di analisi dei segnali discorsivi nell'italiano parlato
}

\section{INTRODUZIONE}

Nel presente contributo osserveremo i diversi ruoli pragmatici assunti dal segnale discorsivo sì nella lingua italiana parlata. È nostro intento servirci di questo esempio concreto per illustrare la pluralità di funzioni e di forze illocutorie di un segnale discorsivo che possono renderne difficoltosa l'elaborazione e l'interpretazione. Il metodo adottato per l'individuazione dei diversi ruoli pragmatici svolti dal segnale prescelto, si propone di costituire un modello di analisi applicabile anche ad altri elementi linguistici aventi simili proprietà pragmatiche.

Le funzioni pragmatiche qui individuate sono già state riscontrate e discusse nei contributi di altri autori che trattano le proprietà di $s \grave{i}$ in qualità di segnale discorsivo $^{1}$ : nel suo contributo sulla pragmatica contrastiva tra i segnali discorsivi nella lingua italiana e nella lingua croata, Tekavčić (1989) si riferisce a sì come a uno dei mezzi linguistici per l'espressione dell'affermazione, della conferma delle parole dell'interlocutore, dell'intensificazione dell'affermazione e dell'equiprobabilità tra l'affermazione e la negazione olofrastica (opposizione tra sì e no) (Tekavčić 1989: 136-137); nel capitolo dedicato alle profrasi, incluso nella Grande grammatica italiana di consultazione, Bernini (1995: 179-182) evidenzia gli usi di sì (e no) che, perdendo la loro caratteristica di proforme, tramite l'acquisizione di altre funzioni di articolazione del discorso, "possono servire e regolare la conversazione» (ibidem: 179); Bazzanella (1995) se ne serve per fornire esempi illustrativi della funzione interattiva per la presa di turno (Bazzanella 1995: 233), per l'espressione di accordo e/o conferma (ibidem : 242), per la ricezione e acquisizione di conoscenza (ibidem : 244) e della funzione metatestuale, ovvero come uno degli esempi di segnali demarcativi (ibidem : 248); Adorno (2007) presenta l'analisi dell'uso di sì (insieme a no e cosi) in apprendenti di italiano L2 residenti in Italia; nella sua tassonomia dei connettivi testuali Samardžić (1995) inserisce sì tra i «connettivi testuali interni» e infine Verdonik (2007: 50-58) svolge un'accurata analisi dei ruoli pragmatici dello sloveno $\mathbf{j a}$, i quali possono essere riscontrati in gran parte anche nei ruoli pragmatici dell'italiano sì.

\footnotetext{
* Indirizzo dell'autrice: Filozofska fakulteta, Oddelek za romanske jezike in književnosti, Aškerčeva 2, 1000 Ljubljana, Slovenia. Email: jana.kenda@amis.net

${ }^{1}$ Per i riferimenti specifici a studi precedentemente svolti, vedi infra la presentazione dei singoli ruoli.
} 


\section{ANALISI}

\subsection{Presentazione del corpus e del metodo di analisi}

La ricerca è stata svolta su un corpus autentico di lingua parlata, con una durata complessiva di 02:48:21 e un totale di 24.968 parole. I diversi dialoghi che compongono il corpus in oggetto sono tratti da tre fonti diverse, distinte come segue: a) corpus Kenda: conversazioni registrate e selezionate dall'autrice (fonte prevalente: la rete televisiva pubblica italiana RAI);

b) corpus LIP: trascrizioni di conversazioni spontanee reperite dal sito che contiene una versione online del corpus LIP (Lessico di frequenza dell'italiano parlato) (cfr. De Mauro, Mancini, Vedovelli, Voghera 1993);

c) corpus C-ORAL-ROM: trascrizioni di conversazioni spontanee della parte italiana del C-ORAL-ROM (cfr. Cresti 2005), in particolare le interazioni informali.

Nell'analisi di sì, si è proceduto adottando il metodo articolato nei seguenti passaggi:

- prima di tutto è stato individuato il numero di occorrenze di questo segnale nel corpus;

- si è poi proceduto all'osservazione del segnale e dei suoi ruoli pragmatici, con l'intento di comprendere come il segnale influisce sul discorso in un contesto attualizzato;

- è stato individuato il numero di occorrenze del segnale in posizione indipendente, iniziale, mediana e finale; la posizione del segnale all'interno dell'enunciato si rivela infatti di vitale importanza per la comprensione della sua funzione pragmatica;

- si è osservata infine l'occorrenza del segnale in concatenazioni con altri segnali (sì beh eh, sì vabbè, sì come no, mah sì però) o altre parole (sì grazie, sì ma) o cumuli (che si realizzano con la ripetizione dello stesso segnale). Da ciò si può dedurre il collegamento tra il segnale e la sua posizione nell'enunciato, nonché arrivare a individuare cumuli fissi.

\subsection{Sì}

Nell'ambito della ricerca all'interno di segnali discorsivi, sì attrae subito l'attenzione perché il suo uso si allontana spesso da quello che è il valore semantico primario. Incuriosisce anche la varietà dei valori che la sua occorrenza produce nel quadro dei contesti attualizzati. Nel nostro corpus viene usato 267 volte, il che rappresenta $1^{‘} 1,06 \%$ di tutte le parole. Il LIP di De Mauro et al. (1993) lo classifica al $24^{\circ}$ posto tra le parole più frequenti nella lingua italiana.

I dizionari monolingui italiani Lo Zingarelli, il Vocabolario della lingua italiana, e il De Mauro Paravia lo classificano come avverbio (con valore affermativo o rafforzativo), sostantivo (il fatidico si), aggettivo invariabile (una giornata sì) e congiunzione (devi far sì che tutto si risolva). Non si fa riferimento, in nessuno dei vocabolari citati, a ruoli pragmatici svolti da questo elemento linguistico. 


\subsubsection{Ruoli pragmatici di sì}

Nella trattazione dei singoli valori pragmatici di sì individuati nel nostro corpus, ogni denominazione è corredata da una breve descrizione (o spiegazione), fatta in base all'osservazione del comportamento del segnale all'interno di contesti attualizzati, e da esempi illustrativi. Si dovrà notare che la delimitazione tra i singoli valori pragmatici individuati non risulta sempre netta e ben determinata.

\section{AFFERMAZIONE OLOFRASTICA (nei significati di «è vero» $e$ "sono d'accordo»)}

Laddove rappresenta la risposta a una domanda, sì è un'affermazione olofrastica equivalente a una proposizione affermativa (può costituire così una parte essenziale del contenuto preposizionale o essere, appunto, una profrase). In questi casi, il suo valore semantico parafrasato potrebbe corrispondere a «è vero»:

16C: Simenon fumava la pipa

$17 C:$ sì

${\text { (CAMILLERI })^{3}}^{3}$

Spesso il parlante non si limita alla sola risposta con sì, ma sottolinea la propria affermazione con altre parole (ad es. ripetendo una parte dell'enunciato precedente) e con questa aggiunta di elementi non solo rafforza il valore affermativo di sì, ma ribadisce l'atteggiamento positivo del parlante nei confronti della comunicazione, come nell'esempio:

104ANG: un parcheggio sotterraneo

105LUI: cioè (-) adesso qua non si vede (-) sì (-) sotterraneo (ARCHI)

\section{Co-occorrenza in concatenazioni o cumuli}

Lo scambio comunicativo che segue contiene due esempi di co-occorrenza con altre espressioni affermative che a loro volta implicano un'attenuazione:

(3) $\quad 267 F$ :

| cioè l'ultima parola che sua nonna disse nella villa di Adriano è stata è una bellezza insostenibile e morì

268C: eh beh sì

269F: è una vita:::

270C:

| insomma una nonna straordinaria una vita:::

271F: | altrettanto

272C: | sì vabbè

(CAMILLERI)

\footnotetext{
${ }^{2}$ In riferimento a questo ruolo pragmatico, è stata ripresa la distinzione tra l'affermazione (pritrjevanje) e il consenso (strinjanje), con i rispettivi valori semantici parafrasati «è vero» (tako je) e «sono d'accordo» (strinjam se) da Verdonik (2007: 50-51).

${ }^{3}$ Questa abbreviazione, come le altre che seguono ciascun esempio, serve a individuare i singoli testi del corpus. L'elenco completo delle abbreviazioni si trova alla fine di questo contributo.
} 
Anche la combinazione dei segnali sì e no, alquanto frequente nella lingua parlata, esprime l'attenuazione di un leggero disaccordo. $S i$ in un certo senso minimizza il disaccordo espresso esplicitamente da no, che lo segue immediatamente. Sì in questo caso ha una forte funzione interattiva, proprio grazie a questa facoltà di minimizzazione:

196C: beh sei già alla quinta edizione prefazione di Beppe Grillo

197OB: sì no forse alla settima ma con il passaparola e questo invece è un passaparola un pochino più allargato (-) riconosciamolo (TUTTOB)

(5) 138TAM: e indo' le mettete / le candele // 'un c'è spazio nemmeno indoe mettere i piatti / si mette le candele

139VER: no / le metti / sì / no vabbè / lì su i' mobilino / a parte è di legno / è un po' pericoloso (FESTASIM)

Oltre ad esprimere la pura affermazione, sì può contenere un'altra sfumatura di significato che rimanda al rapporto tra i due parlanti o alle loro opinioni. Si tratta della situazione di accordo o consenso del secondo parlante con il primo, con il valore semantico di sì che corrisponde a «sono d'accordo»: in questo caso non si tratta di rispondere a domande, ma piuttosto di ribadire l'enunciato dell'altro, scambiare idee, esperienze, proposte:
49F:
| però̀:: però guardi sa cos'è nella mia vita che se dico sì sbaglio se dico no sbaglio se mi scuso strasbaglio
50C: eh
51F: quindi non vorrei trasformarmi ]
$52 C$ :
| faccio io
53F: faccia lei sì lo dica e si scusi ecco
(CAMILLERI)

\section{SEGNALE DI MANTENIMENTO DELL'ATTENZIONE o CONFERMA DELLA RICEZIONE DEL MESSAGGIO ( $\approx$ capisco) ${ }^{4}$}

Questo ruolo pragmatico è riservato esclusivamente all'impiego da parte dell'interlocutore, ossia funge da mezzo espressivo di cui si serve l'interlocutore per offrire un contributo alla conversazione. Infatti in questi casi il significato di sì può essere interpretato con «capisco» ${ }^{5}$ o può essere privo di qualsiasi valore semantico, e usato solo per ribadire la propria presenza nella conversazione e il desiderio di seguire il discorso. L'alta frequenza di questo ruolo pragmatico nella lingua parlata

\footnotetext{
${ }^{4}$ La denominazione di questo valore pragmatico, ripresa da Bernini (1995: 179), è riscontrabile anche nella classificazione di Bazzanella («ricezione e acquisizione di conoscenza», Bazzanella 1995: 244).

${ }^{5}$ Verdonik (2007) attribuisce il valore semantico «capisco» (razumem) all'uso di sì in quanto "conferma della ricezione e dell'attenzione» (potrditev razumevanja in pozornosti) (Verdonik 2007: 52).
} 
è giustificata dal suo valore fatico, di cui l'interlocutore si serve per segnalare periodicamente la propria collaborazione alla conversazione e l'interessamento alle parole altrui, oltre che mettere maggiormente a proprio agio l'altro (nonostante sia molto difficile capire quando questo «segnale di mantenimento dell'attenzione» sia un'espressione sincera e quando invece sia usato per motivi di pura cortesia).

Il proferimento di sì nei seguenti esempi non rappresenta alcuna espressione di conferma, bensì agisce come segno di mantenimento dell'attenzione da parte del parlante:

32C: ma ho detto ma la signora è d'una certa età l'unica cosa che dissi fu

una sciocchezza il'aver aperto la bocca

33F: $\quad$ i sì

34C: disse già (-) ma in gioventù lei pensa che Maigret avrebbe sposato una ragazza così bella (-) poi l'accettò l'accettò perché riuscimmo a invecchiarla bene (-) e mandammo mandammo a lui la fotografia e insomma si persuase (CAMILLERI)

$\begin{array}{ll}\text { 75A: sei uova (-) senti } & \text { I ma intanto che io metto le uova } \\ \text { 76AS: } & \text { | sì }\end{array}$

77A: i bambini (-) cosa possono ballare (-) eh $\uparrow$ (PROVA 1)

\section{CONFERMA ENFATICA ${ }^{6}$}

$S \grave{i}$ viene usato in funzione di segnale di conferma enfatica di quanto si va dicendo: in questi casi vengono messi in particolare rilievo i sintagmi che precedono il segnale sempre in modo diretto. Si noti che in questa funzione sì non viene mai accompagnato da altri segnali, essendo l'enfasi già esplicitata nel collegamento con il sintagma che lo precede.

(9) 6F: e ora devo fare una domanda di avvicinamento così per conoscerci perché non ci siamo mai incontrati (-) solo una volta in collegamento

$7 C$ : in collegamento si

(CAMILLERI)

(10) 274C: no non sono stato un grande amico di Sciascia (-) sono stato un amico di

Sciascia i grandi amici di Sciascia lo chiamavano Nanà

275F: ah ecco la differenza è che i grandi amici lo chiamavano Nanà

276C: Nanà sì

(CAMILLERI)

\section{FUNZIONE DI COLLEGAMENTO}

Accanto alla funzione primaria di affermazione che garantisce una buona intesa tra i partecipanti al processo comunicativo, osserviamo un'importante funzione pragmatica di sì che è quella di collegamento con l'enunciato precedente. La posizione preferenziale per questo ruolo è iniziale, e i casi possono essere diversi: o

${ }^{6}$ Denominazione ripresa da Bernini (1995: 180-181). 
serve da «ponte» tra due enunciati consecutivi dello stesso parlante, interrotti da un enunciato dell'altro parlante, oppure serve da collegamento con l'enunciato precedente dell'altro parlante, oppure, ancora, segue domande che non sono totali.

Si consideri il seguente caso in cui l'apertura del turno tramite sì fa da ponte di collegamento tra gli enunciati dei due parlanti:

162MB: [...] per individuarli per una diagnosi precoce (-) quindi l'attenzione in Europa su questo c'è è I alta

163C: i certo

164MB: e tende a prevenire i disturbi da stress

65BM: sì volevo aggiungere proprio a questo

$166 C$ :

i prego

167BM: mi torna in mente lo stesso studio fatto in Svezia [...]

(TUTTOB)

In questo senso, si noti un confronto con casi analoghi in sloveno: in riferimento a questa funzione Verdonik (2007) esalta il «potere di collegamento» di questo segnale (cfr. Verdonik 2007: 53):
[Tpma27.trs]
K8: eee koliko koliko pa vam pošiljajo to? ker v eni izmed ta velikih dvoran bi moglo biti Amal: ja recimo «dvorana Tabor» nam ne pošilja programa [traduzione]
K8: e:::: quanti quanti ve lo mandano? perché in una tra le sale grandi dovrebbe esserci Amal: sì diciamo la "sala Tabor" non ce lo manda il programma

$S i$ è ancora in funzione di collegamento quando, confermando l'enunciato precedente, funge quasi da elemento di «distacco» dall'oggetto di discussione fino ad allora trattato, dato che il parlante cambia argomento subito dopo il proferimento del segnale. Anche in questo caso l'uso di sì conferma l'attenzione del parlante, ma è più rilevante la sua funzione cataforica di istaurazione del collegamento con ciò che segue. Si noti che, a differenza di questa, tutte le funzioni di sì fino ad ora presentate sono anaforiche:

(13) 74C: mi dice ci sono tre belle ragazze (-) questa (-) dicendomi dei nomi impronunciabili ; che io intanto f-[firmavo] è Miss Svezia e questo significa girare

75F: $\quad$ is sì

76C: il ferro nella piaga lei capisce (-) no $\uparrow$

77F: sì (-) vorrei fare una domanda da fan (-) se posso

(CAMILLERI) 


\section{Co-occorrenza in concatenazioni o cumuli}

Nella sua funzione bivalente di collegamento e distacco, sì viene spesso accompagnato da altri segni che esprimono il «distacco» dall'argomento precedente in modo ancora più evidente (tra i più frequenti segnaliamo soprattutto cioè e però):

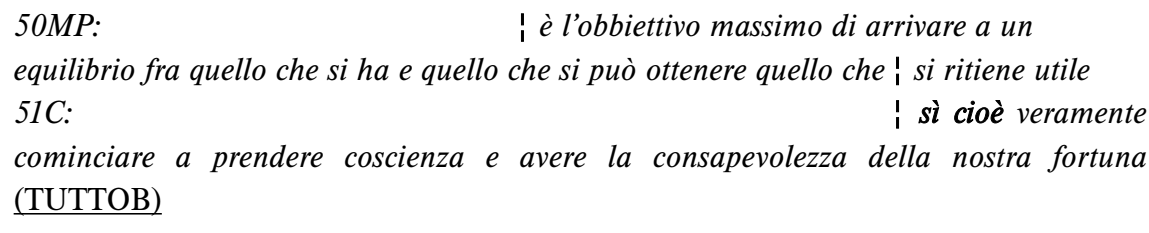

(15) 99E: è la nostra cultura che a volte crea dei tabù dei tabù mentali sul sesso (-) degli anziani Arnaldo 100A: mah sì però va detto e non so se Pasini è d'accordo che il tabù non è una cosa per forza negativa eh (-) (UNOM)

\section{SEGNALE USATO PER GUADAGNARE TEMPO}

Si tratta del caso in cui il parlante usa sì se ha bisogno di guadagnare tempo, il più delle volte per organizzare i propri pensieri, riflettere sulle proprie mosse discorsive;

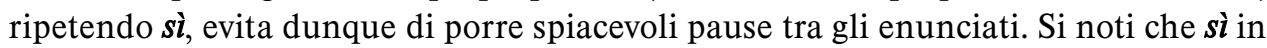
questo ruolo pragmatico viene spesso preceduto e seguito da pause più o meno brevi che apportano un ulteriore guadagno di tempo al parlante in corso, come è evidente dal seguente esempio:

$$
\begin{aligned}
& \text { 219AS: quella è la crema che è crema (-) sì (-) possiamo vedere anche quella se } \\
& \text { vuoi vediamola (PROVA 1) }
\end{aligned}
$$

\section{Co-occorrenza in concatenazioni o cumuli}

Le possibilità di combinazione con sì, quando il parlante è incerto o cerca di guadagnare tempo, sono varie. A volte le diverse concatenazioni si trasformano anche in tic verbali, di cui alcuni parlanti si appropriano ed altri no. Nel seguente scambio comunicativo i segnali sono usati in funzione di riempitivi e se ne può notare l'assoluta assenza di significato:
14VER: condita con l'olio (-) e il sale (-) <normale>
15TAM: sì (-) sì (-) uno poi se la condisce come vole
16VER: eh (-) però questa (-) vabbè (-) sì (-) tipo insieme a (-) i piatti freddi uno la abbina:::
(FESTASIM) 


\section{RUOLI PRAGMATICI DI «Sİ»: Modello di analisi dei segnali discorsivi nell'italiano parlato}

\section{INTRODUZIONE}

Nel presente contributo osserveremo i diversi ruoli pragmatici assunti dal segnale discorsivo sì nella lingua italiana parlata. È nostro intento servirci di questo esempio concreto per illustrare la pluralità di funzioni e di forze illocutorie di un segnale discorsivo che possono renderne difficoltosa l'elaborazione e l'interpretazione. Il metodo adottato per l'individuazione dei diversi ruoli pragmatici svolti dal segnale prescelto, si propone di costituire un modello di analisi applicabile anche ad altri elementi linguistici aventi simili proprietà pragmatiche.

Le funzioni pragmatiche qui individuate sono già state riscontrate e discusse nei contributi di altri autori che trattano le proprietà di $s \grave{i}$ in qualità di segnale discorsivo $^{1}$ : nel suo contributo sulla pragmatica contrastiva tra i segnali discorsivi nella lingua italiana e nella lingua croata, Tekavčić (1989) si riferisce a sì come a uno dei mezzi linguistici per l'espressione dell'affermazione, della conferma delle parole dell'interlocutore, dell'intensificazione dell'affermazione e dell'equiprobabilità tra l'affermazione e la negazione olofrastica (opposizione tra sì e no) (Tekavčić 1989: 136-137); nel capitolo dedicato alle profrasi, incluso nella Grande grammatica italiana di consultazione, Bernini (1995: 179-182) evidenzia gli usi di sì (e no) che, perdendo la loro caratteristica di proforme, tramite l'acquisizione di altre funzioni di articolazione del discorso, "possono servire e regolare la conversazione» (ibidem: 179); Bazzanella (1995) se ne serve per fornire esempi illustrativi della funzione interattiva per la presa di turno (Bazzanella 1995: 233), per l'espressione di accordo e/o conferma (ibidem : 242), per la ricezione e acquisizione di conoscenza (ibidem : 244) e della funzione metatestuale, ovvero come uno degli esempi di segnali demarcativi (ibidem : 248); Adorno (2007) presenta l'analisi dell'uso di sì (insieme a no e cosi) in apprendenti di italiano L2 residenti in Italia; nella sua tassonomia dei connettivi testuali Samardžić (1995) inserisce sì tra i «connettivi testuali interni» e infine Verdonik (2007: 50-58) svolge un'accurata analisi dei ruoli pragmatici dello sloveno $\mathbf{j a}$, i quali possono essere riscontrati in gran parte anche nei ruoli pragmatici dell'italiano sì.

\footnotetext{
* Indirizzo dell'autrice: Filozofska fakulteta, Oddelek za romanske jezike in književnosti, Aškerčeva 2, 1000 Ljubljana, Slovenia. Email: jana.kenda@amis.net

${ }^{1}$ Per i riferimenti specifici a studi precedentemente svolti, vedi infra la presentazione dei singoli ruoli.
} 


\section{ANALISI}

\subsection{Presentazione del corpus e del metodo di analisi}

La ricerca è stata svolta su un corpus autentico di lingua parlata, con una durata complessiva di 02:48:21 e un totale di 24.968 parole. I diversi dialoghi che compongono il corpus in oggetto sono tratti da tre fonti diverse, distinte come segue: a) corpus Kenda: conversazioni registrate e selezionate dall'autrice (fonte prevalente: la rete televisiva pubblica italiana RAI);

b) corpus LIP: trascrizioni di conversazioni spontanee reperite dal sito che contiene una versione online del corpus LIP (Lessico di frequenza dell'italiano parlato) (cfr. De Mauro, Mancini, Vedovelli, Voghera 1993);

c) corpus C-ORAL-ROM: trascrizioni di conversazioni spontanee della parte italiana del C-ORAL-ROM (cfr. Cresti 2005), in particolare le interazioni informali.

Nell'analisi di sì, si è proceduto adottando il metodo articolato nei seguenti passaggi:

- prima di tutto è stato individuato il numero di occorrenze di questo segnale nel corpus;

- si è poi proceduto all'osservazione del segnale e dei suoi ruoli pragmatici, con l'intento di comprendere come il segnale influisce sul discorso in un contesto attualizzato;

- è stato individuato il numero di occorrenze del segnale in posizione indipendente, iniziale, mediana e finale; la posizione del segnale all'interno dell'enunciato si rivela infatti di vitale importanza per la comprensione della sua funzione pragmatica;

- si è osservata infine l'occorrenza del segnale in concatenazioni con altri segnali (sì beh eh, sì vabbè, sì come no, mah sì però) o altre parole (sì grazie, sì ma) o cumuli (che si realizzano con la ripetizione dello stesso segnale). Da ciò si può dedurre il collegamento tra il segnale e la sua posizione nell'enunciato, nonché arrivare a individuare cumuli fissi.

\subsection{Sì}

Nell'ambito della ricerca all'interno di segnali discorsivi, sì attrae subito l'attenzione perché il suo uso si allontana spesso da quello che è il valore semantico primario. Incuriosisce anche la varietà dei valori che la sua occorrenza produce nel quadro dei contesti attualizzati. Nel nostro corpus viene usato 267 volte, il che rappresenta $1^{‘} 1,06 \%$ di tutte le parole. Il LIP di De Mauro et al. (1993) lo classifica al $24^{\circ}$ posto tra le parole più frequenti nella lingua italiana.

I dizionari monolingui italiani Lo Zingarelli, il Vocabolario della lingua italiana, e il De Mauro Paravia lo classificano come avverbio (con valore affermativo o rafforzativo), sostantivo (il fatidico si), aggettivo invariabile (una giornata sì) e congiunzione (devi far sì che tutto si risolva). Non si fa riferimento, in nessuno dei vocabolari citati, a ruoli pragmatici svolti da questo elemento linguistico. 


\subsubsection{Ruoli pragmatici di sì}

Nella trattazione dei singoli valori pragmatici di sì individuati nel nostro corpus, ogni denominazione è corredata da una breve descrizione (o spiegazione), fatta in base all'osservazione del comportamento del segnale all'interno di contesti attualizzati, e da esempi illustrativi. Si dovrà notare che la delimitazione tra i singoli valori pragmatici individuati non risulta sempre netta e ben determinata.

\section{AFFERMAZIONE OLOFRASTICA (nei significati di «è vero» $e$ "sono d'accordo»)}

Laddove rappresenta la risposta a una domanda, sì è un'affermazione olofrastica equivalente a una proposizione affermativa (può costituire così una parte essenziale del contenuto preposizionale o essere, appunto, una profrase). In questi casi, il suo valore semantico parafrasato potrebbe corrispondere a «è vero»:

16C: Simenon fumava la pipa

$17 C:$ sì

${\text { (CAMILLERI })^{3}}^{3}$

Spesso il parlante non si limita alla sola risposta con sì, ma sottolinea la propria affermazione con altre parole (ad es. ripetendo una parte dell'enunciato precedente) e con questa aggiunta di elementi non solo rafforza il valore affermativo di sì, ma ribadisce l'atteggiamento positivo del parlante nei confronti della comunicazione, come nell'esempio:

104ANG: un parcheggio sotterraneo

105LUI: cioè (-) adesso qua non si vede (-) sì (-) sotterraneo (ARCHI)

\section{Co-occorrenza in concatenazioni o cumuli}

Lo scambio comunicativo che segue contiene due esempi di co-occorrenza con altre espressioni affermative che a loro volta implicano un'attenuazione:

(3) $\quad 267 F$ :

| cioè l'ultima parola che sua nonna disse nella villa di Adriano è stata è una bellezza insostenibile e morì

268C: eh beh sì

269F: è una vita:::

270C:

| insomma una nonna straordinaria una vita:::

271F: | altrettanto

272C: | sì vabbè

(CAMILLERI)

\footnotetext{
${ }^{2}$ In riferimento a questo ruolo pragmatico, è stata ripresa la distinzione tra l'affermazione (pritrjevanje) e il consenso (strinjanje), con i rispettivi valori semantici parafrasati «è vero» (tako je) e «sono d'accordo» (strinjam se) da Verdonik (2007: 50-51).

${ }^{3}$ Questa abbreviazione, come le altre che seguono ciascun esempio, serve a individuare i singoli testi del corpus. L'elenco completo delle abbreviazioni si trova alla fine di questo contributo.
} 
Anche la combinazione dei segnali sì e no, alquanto frequente nella lingua parlata, esprime l'attenuazione di un leggero disaccordo. $S i$ in un certo senso minimizza il disaccordo espresso esplicitamente da no, che lo segue immediatamente. Sì in questo caso ha una forte funzione interattiva, proprio grazie a questa facoltà di minimizzazione:

196C: beh sei già alla quinta edizione prefazione di Beppe Grillo

197OB: sì no forse alla settima ma con il passaparola e questo invece è un passaparola un pochino più allargato (-) riconosciamolo (TUTTOB)

(5) 138TAM: e indo' le mettete / le candele // 'un c'è spazio nemmeno indoe mettere i piatti / si mette le candele

139VER: no / le metti / sì / no vabbè / lì su i' mobilino / a parte è di legno / è un po' pericoloso (FESTASIM)

Oltre ad esprimere la pura affermazione, sì può contenere un'altra sfumatura di significato che rimanda al rapporto tra i due parlanti o alle loro opinioni. Si tratta della situazione di accordo o consenso del secondo parlante con il primo, con il valore semantico di sì che corrisponde a «sono d'accordo»: in questo caso non si tratta di rispondere a domande, ma piuttosto di ribadire l'enunciato dell'altro, scambiare idee, esperienze, proposte:
49F:
| però̀:: però guardi sa cos'è nella mia vita che se dico sì sbaglio se dico no sbaglio se mi scuso strasbaglio
50C: eh
51F: quindi non vorrei trasformarmi ]
$52 C$ :
| faccio io
53F: faccia lei sì lo dica e si scusi ecco
(CAMILLERI)

\section{SEGNALE DI MANTENIMENTO DELL'ATTENZIONE o CONFERMA DELLA RICEZIONE DEL MESSAGGIO ( $\approx$ capisco) ${ }^{4}$}

Questo ruolo pragmatico è riservato esclusivamente all'impiego da parte dell'interlocutore, ossia funge da mezzo espressivo di cui si serve l'interlocutore per offrire un contributo alla conversazione. Infatti in questi casi il significato di sì può essere interpretato con «capisco» ${ }^{5}$ o può essere privo di qualsiasi valore semantico, e usato solo per ribadire la propria presenza nella conversazione e il desiderio di seguire il discorso. L'alta frequenza di questo ruolo pragmatico nella lingua parlata

\footnotetext{
${ }^{4}$ La denominazione di questo valore pragmatico, ripresa da Bernini (1995: 179), è riscontrabile anche nella classificazione di Bazzanella («ricezione e acquisizione di conoscenza», Bazzanella 1995: 244).

${ }^{5}$ Verdonik (2007) attribuisce il valore semantico «capisco» (razumem) all'uso di sì in quanto "conferma della ricezione e dell'attenzione» (potrditev razumevanja in pozornosti) (Verdonik 2007: 52).
} 
è giustificata dal suo valore fatico, di cui l'interlocutore si serve per segnalare periodicamente la propria collaborazione alla conversazione e l'interessamento alle parole altrui, oltre che mettere maggiormente a proprio agio l'altro (nonostante sia molto difficile capire quando questo «segnale di mantenimento dell'attenzione» sia un'espressione sincera e quando invece sia usato per motivi di pura cortesia).

Il proferimento di sì nei seguenti esempi non rappresenta alcuna espressione di conferma, bensì agisce come segno di mantenimento dell'attenzione da parte del parlante:

32C: ma ho detto ma la signora è d'una certa età l'unica cosa che dissi fu

una sciocchezza il'aver aperto la bocca

33F: $\quad$ i sì

34C: disse già (-) ma in gioventù lei pensa che Maigret avrebbe sposato una ragazza così bella (-) poi l'accettò l'accettò perché riuscimmo a invecchiarla bene (-) e mandammo mandammo a lui la fotografia e insomma si persuase (CAMILLERI)

$\begin{array}{ll}\text { 75A: sei uova (-) senti } & \text { I ma intanto che io metto le uova } \\ \text { 76AS: } & \text { | sì }\end{array}$

77A: i bambini (-) cosa possono ballare (-) eh $\uparrow$ (PROVA 1)

\section{CONFERMA ENFATICA ${ }^{6}$}

$S \grave{i}$ viene usato in funzione di segnale di conferma enfatica di quanto si va dicendo: in questi casi vengono messi in particolare rilievo i sintagmi che precedono il segnale sempre in modo diretto. Si noti che in questa funzione sì non viene mai accompagnato da altri segnali, essendo l'enfasi già esplicitata nel collegamento con il sintagma che lo precede.

(9) 6F: e ora devo fare una domanda di avvicinamento così per conoscerci perché non ci siamo mai incontrati (-) solo una volta in collegamento

$7 C$ : in collegamento si

(CAMILLERI)

(10) 274C: no non sono stato un grande amico di Sciascia (-) sono stato un amico di

Sciascia i grandi amici di Sciascia lo chiamavano Nanà

275F: ah ecco la differenza è che i grandi amici lo chiamavano Nanà

276C: Nanà sì

(CAMILLERI)

\section{FUNZIONE DI COLLEGAMENTO}

Accanto alla funzione primaria di affermazione che garantisce una buona intesa tra i partecipanti al processo comunicativo, osserviamo un'importante funzione pragmatica di sì che è quella di collegamento con l'enunciato precedente. La posizione preferenziale per questo ruolo è iniziale, e i casi possono essere diversi: o

${ }^{6}$ Denominazione ripresa da Bernini (1995: 180-181). 
serve da «ponte» tra due enunciati consecutivi dello stesso parlante, interrotti da un enunciato dell'altro parlante, oppure serve da collegamento con l'enunciato precedente dell'altro parlante, oppure, ancora, segue domande che non sono totali.

Si consideri il seguente caso in cui l'apertura del turno tramite sì fa da ponte di collegamento tra gli enunciati dei due parlanti:

162MB: [...] per individuarli per una diagnosi precoce (-) quindi l'attenzione in Europa su questo c'è è I alta

163C: i certo

164MB: e tende a prevenire i disturbi da stress

65BM: sì volevo aggiungere proprio a questo

$166 C$ :

i prego

167BM: mi torna in mente lo stesso studio fatto in Svezia [...]

(TUTTOB)

In questo senso, si noti un confronto con casi analoghi in sloveno: in riferimento a questa funzione Verdonik (2007) esalta il «potere di collegamento» di questo segnale (cfr. Verdonik 2007: 53):
[Tpma27.trs]
K8: eee koliko koliko pa vam pošiljajo to? ker v eni izmed ta velikih dvoran bi moglo biti Amal: ja recimo «dvorana Tabor» nam ne pošilja programa [traduzione]
K8: e:::: quanti quanti ve lo mandano? perché in una tra le sale grandi dovrebbe esserci Amal: sì diciamo la "sala Tabor" non ce lo manda il programma

$S i$ è ancora in funzione di collegamento quando, confermando l'enunciato precedente, funge quasi da elemento di «distacco» dall'oggetto di discussione fino ad allora trattato, dato che il parlante cambia argomento subito dopo il proferimento del segnale. Anche in questo caso l'uso di sì conferma l'attenzione del parlante, ma è più rilevante la sua funzione cataforica di istaurazione del collegamento con ciò che segue. Si noti che, a differenza di questa, tutte le funzioni di sì fino ad ora presentate sono anaforiche:

(13) 74C: mi dice ci sono tre belle ragazze (-) questa (-) dicendomi dei nomi impronunciabili ; che io intanto f-[firmavo] è Miss Svezia e questo significa girare

75F: $\quad$ is sì

76C: il ferro nella piaga lei capisce (-) no $\uparrow$

77F: sì (-) vorrei fare una domanda da fan (-) se posso

(CAMILLERI) 


\section{Co-occorrenza in concatenazioni o cumuli}

Nella sua funzione bivalente di collegamento e distacco, sì viene spesso accompagnato da altri segni che esprimono il «distacco» dall'argomento precedente in modo ancora più evidente (tra i più frequenti segnaliamo soprattutto cioè e però):

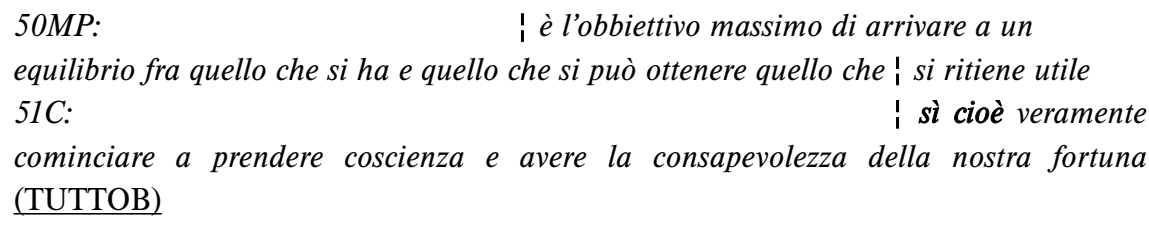

(15) 99E: è la nostra cultura che a volte crea dei tabù dei tabù mentali sul sesso (-) degli anziani Arnaldo 100A: mah sì però va detto e non so se Pasini è d'accordo che il tabù non è una cosa per forza negativa eh (-) (UNOM)

\section{SEGNALE USATO PER GUADAGNARE TEMPO}

Si tratta del caso in cui il parlante usa sì se ha bisogno di guadagnare tempo, il più delle volte per organizzare i propri pensieri, riflettere sulle proprie mosse discorsive;

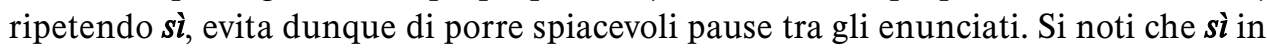
questo ruolo pragmatico viene spesso preceduto e seguito da pause più o meno brevi che apportano un ulteriore guadagno di tempo al parlante in corso, come è evidente dal seguente esempio:

$$
\begin{aligned}
& \text { 219AS: quella è la crema che è crema (-) sì (-) possiamo vedere anche quella se } \\
& \text { vuoi vediamola (PROVA 1) }
\end{aligned}
$$

\section{Co-occorrenza in concatenazioni o cumuli}

Le possibilità di combinazione con sì, quando il parlante è incerto o cerca di guadagnare tempo, sono varie. A volte le diverse concatenazioni si trasformano anche in tic verbali, di cui alcuni parlanti si appropriano ed altri no. Nel seguente scambio comunicativo i segnali sono usati in funzione di riempitivi e se ne può notare l'assoluta assenza di significato:
14VER: condita con l'olio (-) e il sale (-) <normale>
15TAM: sì (-) sì (-) uno poi se la condisce come vole
16VER: eh (-) però questa (-) vabbè (-) sì (-) tipo insieme a (-) i piatti freddi uno la abbina:::
(FESTASIM) 


\section{ESPRESSIONE DI COMPARTECIPAZIONE}

La sola parolina sì (in questa funzione spesso pronunciata con la vocale allungata), nonostante la sua brevità e semplicità, può implicare addirittura compartecipazione interessata, incredulità o sorpresa rispetto all'enunciato dell'interlocutore. Si osservi che questo ruolo è fortemente marcato dall'intonazione alta-ascendente (cfr. Bernini 1995: 181) che è stata riscontrata in tutti gli esempi reperiti nel corpus qui considerato.

In questi casi, la funzione interattiva, cioè fatica di sì, raggiunge il massimo della sua espressività, essendo la sua comparsa dipendente e giustificabile sempre e soltanto dall'enunciato dell'altro parlante. Segnaliamo che per questa funzione la co-occorrenza in concatenazioni o cumuli non è possibile.

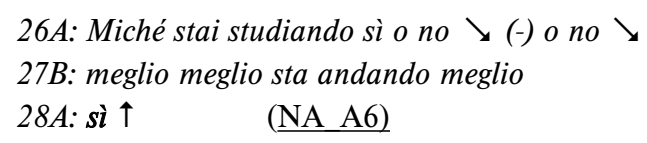

93A: la macchina andava bene la macchina sì $\uparrow$

94B: sì sì stasera cambiamo

95A: si::: $\uparrow \quad$ (NA A6)

\section{OPPOSIZIONE Sİ vS NO}

Si viene spesso opposto a no, soprattutto quando entrambi fungono da profrasi: sono casi in cui il parlante necessita di evidenziare gli elementi che compongono i due poli opposti o esprime la richiesta perentoria di una scelta o di una risposta definitiva. Ne sono una dimostrazione i seguenti esempi illustrativi:

148M: non abbiamo niente su Pinocchio $\uparrow$

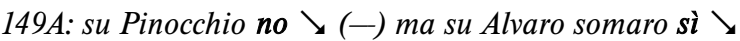

(PROVA 1)

(21) 202E: senti Gisella ma ti è mai capitato di dare invece un consiglio a una coppia giovane::

203GS: ma no perché io non li do mai (-) quando me li chiedono sì ma non è che me ne chiedano tanti

(UNOM)

\section{SEGNALE DI (AUTO)CORREZIONE}

Sì rappresenta solo uno tra i diversi segnali discorsivi per la realizzazione di questa funzione metatestuale, funzione con cui si marcano le correzioni introducendo parafrasi, esemplificazioni o semplicemente correzioni di ciò che il parlante ha proferito in precedenza. È frequente l'abbinamento di sì con altri segnali discorsivi il cui valore semantico determina in forma più esplicita il tipo di (auto)correzione prescelto dal parlante, ad esempio sì ma per una precisazione, sì voglio dire per la parafrasi, sì cioè per la correzione, sì ecco, sì per esempio per l'esemplificazione 
(cfr. Bazzanella 1996: 248-249). Nelle due illustrazioni seguenti forniamo esempi di correzione (22) e precisazione (23):

109B: ah ci hai pure::: i numeri memorizzati tutti

110C: sì ah no questo è comodo perché ci ha::: l'antennina eh che non la devi estrarre (RM_A2)

(23) 185GS: ed è tutto una cosa di tenerezza infinita (-) c'è questo c'è amore sì passione sì ma è questo (-) l'intimità enorme che c'è (-) quella è la cosa importante

(UNOM)

\section{FUNZIONE DI MODULAZIONE7}

A volte c'è la necessità di minimizzare quanto contenuto nell'enunciato precedente, come se si volesse apparire più modesti, con un atteggiamento che invita a non «esagerare nell'apprezzare». Si parla, in questi casi, della funzione di modulazione del segnale discorsivo che può essere realizzata anche da sì, prevalentemente seguito dal segnale insomma, come illustrato dal seguente esempio:

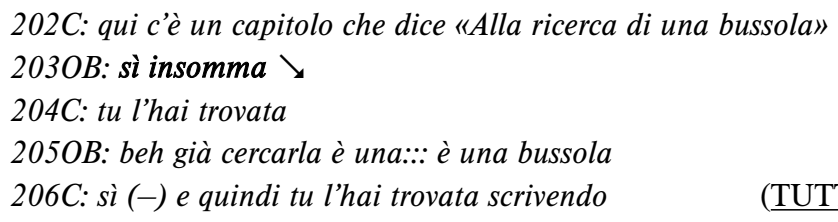

(TUTTOB)

\section{$\mathrm{X}$ RICHIESTA DI CONFERMA ${ }^{8}$}

Mezzo comunicativo proprio del parlante in corso (e indirizzato ovviamente ad almeno un interlocutore), sì? pronunciato in tono ascendente funge come richiesta di conferma delle proprie parole. Questa funzione è solitamente svolta da no? (sempre in tono ascendente), ma un esempio tratto dal corpus dimostrerà come sia possibile raggiungere lo stesso scopo anche con sì. Si noti che la posizione riservata a questa funzione è rigorosamente finale:

\section{(ESEMPI ILLUSTRATIVI DI no)}

(25) 107A: ma và che siete dei bei tipi eh noi stiamo mettendo il limone sulla mela e lo zucchero no $\uparrow$

108AS: limone e lo zucchero sì

(PROVA 1)

(26) 33BV: allora (-) anche Ira Fürstenberg è stata una grande protagonista nella:: è stata e lo è insomma della così della vita 34IF: I mondana principesca no $\uparrow$
i beh
(SORAYA)

\footnotetext{
${ }^{7}$ Tra i diversi tipi di funzioni interattive, Bazzanella (1996) riconose ai segnali discorsivi la facoltà di fungere da «meccanismi di modulazione» impiegati per mitigare (o rafforzare) il contenuto proposizionale di un enunciato (cfr. Bazzanella 1996: 238).

${ }^{8}$ Denominazione ripresa da Bazzanella (1996: 240).
} 


\section{(ESEMPIO ILLUSTRATIVO DI si)}

(27) 93A: la macchina andava bene la macchina si $\uparrow$

94B: sì sì stasera cambiamo ～(NA_A6)

\section{SÌ DI APERTURA}

Nel nostro corpus abbiamo individuato solo un esempio del cosiddetto sì di apertura (o esortativo) che serve per prendere il turno:

\section{COA: sì mh (-) dunque (-) solo una cosa (-) scusate se (-) mi sono assentato (QUART)}

Nonostante il nostro corpus non ne presenti alcun caso illustrativo, ricordiamo una funzione che può essere intesa come una delle possibili varianti del «sì di apertura», cioè quella con cui si esprime la disponibilità alla comunicazione da parte del parlante, come risposta alla chiamata al telefono (Sì, pronto?!) o reazione all'apparizione di una persona in un luogo pubblico o in un esercizio commerciale (reception o portineria di un'azienda o di un albergo, in un negozio), con il valore semantico di «che cosa desidera?» (cfr. Bernini 1995: 180).

\subsubsection{Posizione di sì all'interno dell'enunciato}

Vediamo ora lo schema illustrativo delle posizioni di sì nel nostro corpus. Dall'analisi risulta che nella maggior parte delle occorrenze (i dati statistici dimostrano che si tratta quasi del 50\% dei casi) sì occupa la posizione iniziale, come apertura di replica, mentre nelle altre occorrenze si distribuisce tra la posizione indipendente (in enunciato indipendente), la posizione mediana e quella finale.

\begin{tabular}{|l|c|c|}
\hline \multicolumn{1}{|c|}{ Sİ -267 occorrenze } & $\mathbf{n}^{\circ}$ occorrenze & percentuale rispetto al totale \\
\hline enunciato indipendente & 83 & $31 \%$ \\
\hline posizione iniziale & 133 & $49,8 \%$ \\
\hline posizione mediana & 31 & $11,6 \%$ \\
\hline posizione finale & 20 & $7,5 \%$ \\
\hline
\end{tabular}

Tab.1: Posizione di sì

Solo 21 volte (tra cui: 16 in posizione mediana, 4 iniziale, 1 finale e 0 in enunciato indipendente), che costituiscono il 7,86\% di tutte le occorrenze, sì viene usato dalla parte del parlante, mentre nel restante $92,14 \%$ dei casi viene impiegato dalla parte dell'interlocutore, cioè come un tipo di replica o reazione interattiva nel quadro di uno scambio comunicativo. Come abbiamo avuto modo di precisare già in precedenza, sì è un segnale di accordo per eccellenza, usato in primo luogo con lo scopo di confermare, in secondo di collegare gli enunciati nella comunicazione, perciò è chiara la ragione per cui lo riscontriamo soprattutto in enunciati proferiti 
dall'interlocutore. Ne scaturisce la sua funzione principalmente interattiva (o interazionale) di cui i partecipanti all'atto comunicativo si servono per garantire un valido e positivo andamento alla comunicazione. La sua funzione metatestuale è di importanza notevolmente minore rispetto a quella interattiva, anche se non mancano esempi in cui viene usato in funzione demarcativa (es. (29)) e in funzione di indicatore di riformulazione (es. (30)):

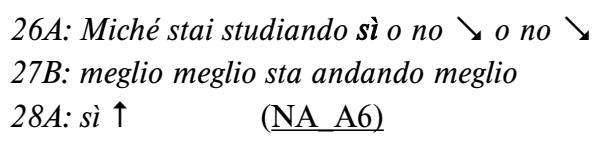

(30) 109B: ah ci hai pure::: i numeri memorizzati tutti

110C: sì ah no questo è comodo perché ci ha::: l'antennina eh che non la devi estrarre (RM_A2)

\section{CONCLUSIONE}

L'analisi svolta sul nostro corpus ha dimostrato che il segnale in questione ricopre prevalentemente ruoli pragmatici e spesso non siamo in grado di fornire una soddisfacente espressione descrittiva del suo valore semantico, soprattutto quando viene usato come profrase (il suo valore semantico dipende di volta in volta dal contesto in cui è usato) o quando svolge funzione di collegamento, funzione di modulazione, stacco/passaggio da una tematica all'altra, o ancora, quando introduce parafrasi o chiarimenti.

In base all'analisi dei diversi ruoli individuati, riassumiamo le principali funzioni svolte dal segnale in questione: i) serve a collegare le singole unità della conversazione (con riferimenti sia anaforici che cataforici), ii) aiuta a stabilire e mantenere diversi tipi di rapporto tra i parlanti, iii) rende possibile l'espressione dell'atteggiamento del parlante nei confronti del contenuto della conversazione e iv) agevola l'organizzazione della conversazione. Osserviamo queste funzioni più dettagliatamente.

i) Nei casi in cui sì svolge il ruolo di collegamento tra le singole unità della conversazione, i riferimenti sono anaforici soprattutto quando costituisce una profrase (infatti la funzione testuale principale, a cui tutti gli usi delle profrasi possono essere ricondotti, è quella di replica e la replica ha sempre un antecedente a cui si riferisce e che è contenuto in un enunciato precedente; cfr. Bernini 1995: 177) e quando viene adoperato dal parlante come collegamento con un enunciato proferito in precedenza, come conferma delle parole altrui o come conferma enfatica. Sono cataforici i collegamenti negli altri casi. Fa eccezione però l'ancoraggio creato dall'uso del ruolo pragmatico di «collegamento e distacco» di sì (il ruolo IV), che incorpora in sé sia il riferimento all'enunciato precedente, sia l'anticipazione di quello seguente.

ii) $S i$ contribuisce all'istaurazione e al mantenimento di diversi tipi di rapporto tra i partecipanti alla comunicazione, da quelli amichevoli e cooperativi, a rapporti contrassegnati dalla contrarietà o obiezione nonché disuguaglianza ossia supremazia 
di uno degli interlocutori. Le situazioni riscontrate illustrano prevalentemente casi in cui il rapporto tra gli interlocutori è piacevole, il che non sorprende data la tendenza dei parlanti a mantenere un clima positivo e amichevole in scambi comunicativi faccia a faccia (si noti che la stessa analisi, svolta su un corpus di conversazioni conflittuali, avrebbe ovviamente un esito del tutto diverso). Ciò nonostante, osserviamo che il segnale analizzato può soddisfare anche le esigenze di espressione di disaccordo o contrarietà (che però, nei casi riscontrati nel nostro corpus, non ha mai assunto toni particolarmente duri).

iii) Uno dei principali impieghi dei segnali discorsivi volti all'espressione dell'atteggiamento del parlante nei confronti del contenuto della conversazione (o nei confronti del contenuto del turno dell'altro interlocutore) viene realizzato da sì nei ruoli di affermazione olofrastica, conferma enfatica, funzione di collegamento e distacco, funzione di compartecipazione e funzione di modulazione.

iv) Per quanto riguarda il suo contributo all'organizzazione della conversazione, è stato rilevato che sì agisce su due campi diversi: da un lato segna il cambio di turno fra i due interlocutori (per stabilire il contatto, per prendere o cedere la parola, per aiutare a non creare pause inutili), dall'altro lato, organizza il contenuto del discorso, sia all'interno dello stesso turno sia tra turni diversi, per connettere le tematiche della conversazione, per limitare il contrasto o disaccordo con l'interlocutore (o come mezzo di attenuazione delle posizioni espresse) e per indicare diverse svolte nella conversazione (come le autocorrezioni o spiegazioni aggiuntive).

\section{CORPUS (abbreviazioni):}

(CAMILLERI); (TUTTOB); (PROVA 1); (UNOM); (SORAYA): dialoghi e conversazioni dal corpus Kenda

(FESTASIM); (QUART): trascrizioni dal corpus C-ORAL-ROM.

(NA A6); (RM A2): trascrizioni dal corpus LIP.

\section{Bibliografia:}

ADorno, Cecilia Maria (2007) «Apprendere il lessico: elaborazione di segnali discorsivi (SI', NO, COSI').» In: M. Chini/P. Desideri/M. E. Favilla/G. Pallotti et al. (a cura di), Atti del VI Congresso internazionale dell'AitLA. Perugia: Guerra Edizioni, 95-121.

BAZZANella, Carla (1995) «I segnali discorsivi.» In: Lorenzo Renzi et al. (a cura di), 225-257. BERNINI, Giuliano (1995) «Le profrasi.» In: Lorenzo Renzi et al. (a cura di), 175-222.

Cresti, Emanuela/Massimo Moneglia (a cura di) (2005) C-ORAL-ROM, Integrated Reference Corpora for Spoken Romance Languages. Amsterdam: John Benjamins Publishing Company.

De Mauro, Tullio/Federico Mancini/Massimo Vedovelli/Miriam Voghera (1993) LIP: Lessico di frequenza dell'italiano parlato. Milano: ETASLIBRI. 
Renzi, Lorenzo/Giampaolo SAlvi/Anna CARDinaletTi (a cura di) (1995) Grande grammatica di consultazione, vol. III. Bologna: Il Mulino.

VERDONIK, Darinka (2007) Jezikovni elementi spontanosti v pogovoru. Maribor: Slavistično društvo.

\section{Povzetek}

\section{PRAGMATIČNE VLOGE »Sİ«:}

Model analize diskurznih označevalcev v govorjenem italijanskem jeziku

Avtorica se ukvarja z opazovanjem in analizo italijanskega diskurznega označevalca sì in različnih vlog, ki jih ta opravlja v govorjenem jeziku.

Predstavljeni so metoda dela, potek analize in kratek oris ugotovljenih pragmatičnih vlog tega jezikovnega elementa na izbranem korpusu ter nekaj zgledov za vsako vlogo posebej. Ugotovljeno je bilo, da se sì pojavlja v naslednjih pragmatičnih vlogah: I/ kot holofrastična pritrditev (s semantičnima vrednostma »tako je« in »strinjam se«); II/ kot signal za vzdrževanje pozornosti ali potrditev razumevanja sporočila (s semantično vrednostjo »razumem«); III/ kot signal za emfatično potrditev; IV/ kot označevalec s povezovalno in »ločevalno« funkcijo; V/ kot signal za pridobivanje časa; VI/ kot signal za izražanje soudeležbe; VII/ kot signal v opoziciji z označevalcem no; VIII/ kot signal za (samo)popravljanje; IX/ kot signal z modulacijsko funkcijo; X/ kot signal zahteve po potrjevanju in XI/ kot otvoritveni sì.

Izhodišče za obravnavo in določanje posameznih vlog temelji na pragmalingvističnih raziskavah, opravljenih v italijanskem in slovenskem jezikoslovju zadnjih let (Bazzanella 1995, Bernini 1995, Adorno 2007; Verdonik 2007). 